\title{
Vitamin D status of British African-Caribbean residents: analysis of the UK Biobank cohort
}

Vitamin D deficiency is a global health issue ${ }^{(1)}$. However, little is known about the vitamin D status of the United Kingdom (UK) African-Caribbean (AC) population, even though this population group is likely at increased risk of vitamin D deficiency, due to latitude and skin type ${ }^{(1,2)}$.

This cross-sectional study explored the $25(\mathrm{OH}) \mathrm{D}$ concentration and vitamin D intake of AC individuals from the UK Biobank Cohort, who were aged 40 years and over at baseline (2006-2010). For context, the AC population was also compared to the African (AF), South Asian (SA) and White European (WE) subsets of the UK Biobank.

For AC, $n=4046$ had a valid 25(OH)D measurement. Of these, $n=1499(37.0 \%)$ were deficient $(<25 \mathrm{nmol} / \mathrm{L}), \mathrm{n}=1906(41.1 \%)$ were insufficient $(25-50 \mathrm{nmol} / \mathrm{L})$ and $\mathrm{n}=642(15.9 \%)$ were sufficient $(>50 \mathrm{nmol} / \mathrm{L})$. Median (IQR) for 25(OH)D was 30.0 (20.9) $\mathrm{nmol} / \mathrm{L}$ and median vitamin $\mathrm{D}$ intake was $1.6(2.6) \mu \mathrm{g} /$ day, with only $\mathrm{n}=64(4.8 \%)$ of AC meeting the UK recommended nutrient vitamin $\mathrm{D}$ intake of $10 \mu \mathrm{g} / \mathrm{day}$. For the other ethnic groups, the median (IQR) for $25(\mathrm{OH}) \mathrm{D}$ concentration was $30.2(20.0) \mathrm{nmol} / \mathrm{L}$ in AF, 20.7 (18.5) nmol/L in SA and 49.2 (29.5) nmol/L in WE. Logistic regression showed that brown/black skin type (OR 1.77, 95\% CI 1.19,2.63), winter blood draw (OR 1.22, 95\% CI 0.98, 1.51), not consuming oily fish (OR 1.74, 95\% CI 1.19, 2.54) and not using vitamin D supplements (OR 2.97, 95\% CI 2.50, 3.53) were predictors of increased odds of vitamin D deficiency $(<25 \mathrm{nmol} / \mathrm{L})$, whilst older age (OR $0.47,95 \% 0.37,0.61)$ and summer (OR $0.36,95 \%$ CI $0.28,0.45)$ or autumn blood draws $(\mathrm{OR}$ $0.63,95 \%$ CI $0.51,0.79$ ) were significantly associated with a reduction in the odds of vitamin $\mathrm{D}$ deficiency.

We found high levels of vitamin D deficiency and insufficiency in this AC population. This is of concern due to the individual and societal implications of an increased morbidity. Of note, the UK Biobank participants may be healthier than the national average ${ }^{(3)}$, meaning these results may be an underestimate of the true degree of vitamin D deficiency in the UK AC population. Public health messaging for this group may need to focus on year-round vitamin D supplementation and increasing intakes of culturally appropriate vitamin $\mathrm{D}$ rich foods. These data also lend support to the urgent requirement for a revised vitamin D RNI for ethnic groups.

\section{Acknowledgments}

This work is part of the $\mathrm{PhD}$ of RM. Vearing, which is funded by the Universities Global Partnership Network, co-supervised by the Universities of Surrey and Wollongong. This research was conducted using the UK Biobank resource under Project 15168 . KC, KH and YP did not view the individual participant data.

\section{References}

1. Wilson LR, Tripkovic L, Hart KH et al. (2017)Proc Nutr Soc, 76(3), 392-399.

2. Clemens TL, Adams JS, Henderson SL et al. (1982) Lancet, 1(8263), 74-76.

3. Fry A, Littlejohns TL, Sudlow C et al. (2017) Am J Epidemiol, 186(9), 1026-1034. 